\title{
Species identification and delineation of pathogenic Mucorales by matrix-assisted laser desorption ionization- time-of-flight mass spectrometry
}

\author{
Jin Shao ${ }^{1,2,3}$, Zhe Wan ${ }^{1,2,3}$, Ruoyu Li $i^{1,2,3}$, JinYu' ${ }^{1,2,3 \#}$ \\ 1Department of Dermatology, Peking University First Hospital, Beijing 100034, China, \\ ${ }^{2}$ Beijing Key Laboratory of Molecular Diagnosis on Dermatoses, Beijing 100034, China, \\ ${ }^{3}$ Research Center for Medical Mycology, Peking University, Beijing 100034, China \\ Running Title: pathogenic Mucorales \\ \# Address correspondence to Jin Yu, yujin676@126.com.
}

\section{Abstract}

Objectives: This study aimed to validate the effectiveness of matrix-assisted laser desorption ionization-time-of-flight mass spectrometry (MALDI-TOF MS)-based identification of filamentous fungi of the order Mucorales.

Methods: A total of 111 clinical strains covering 6 genera preserved at the Research Center for Medical Mycology of Peking University were selected for MALDI-TOF MS analysis. We emphasized the study of 23 strains of Mucor irregularis predominantly isolated from patients in China. We first used the Bruker Filamentous Fungi library v1.0 to identify all 111 isolates. To increase the identification rate, we created a compensatory in-house database, the Beijing Medical University (BMU) database, using 13 reference strains covering 6 species, including M. irregularis, Mucor hiemalis, Mucor racemosus, Cunninghamella bertholletiae, Cunninghamella phaeospora and Cunninghamella echinulata. All 111 isolates were then identified by MALDI-TOF MS using a combination of the Bruker library and BMU database. We also compared phylogenetic trees based on ITS sequence of Rhizopus spp. and Mucor spp. with the MSP dendrograms based on MALDI-TOF MS analysis.

Results: MALDI-TOF MS identified 55 (49.5\%) and 74 (66.7\%) isolates at the species and genus levels, respectively, using the Bruker Filamentous Fungi library v1.0 alone. A combination of the Bruker library and BMU database allowed MALDI-TOF MS to

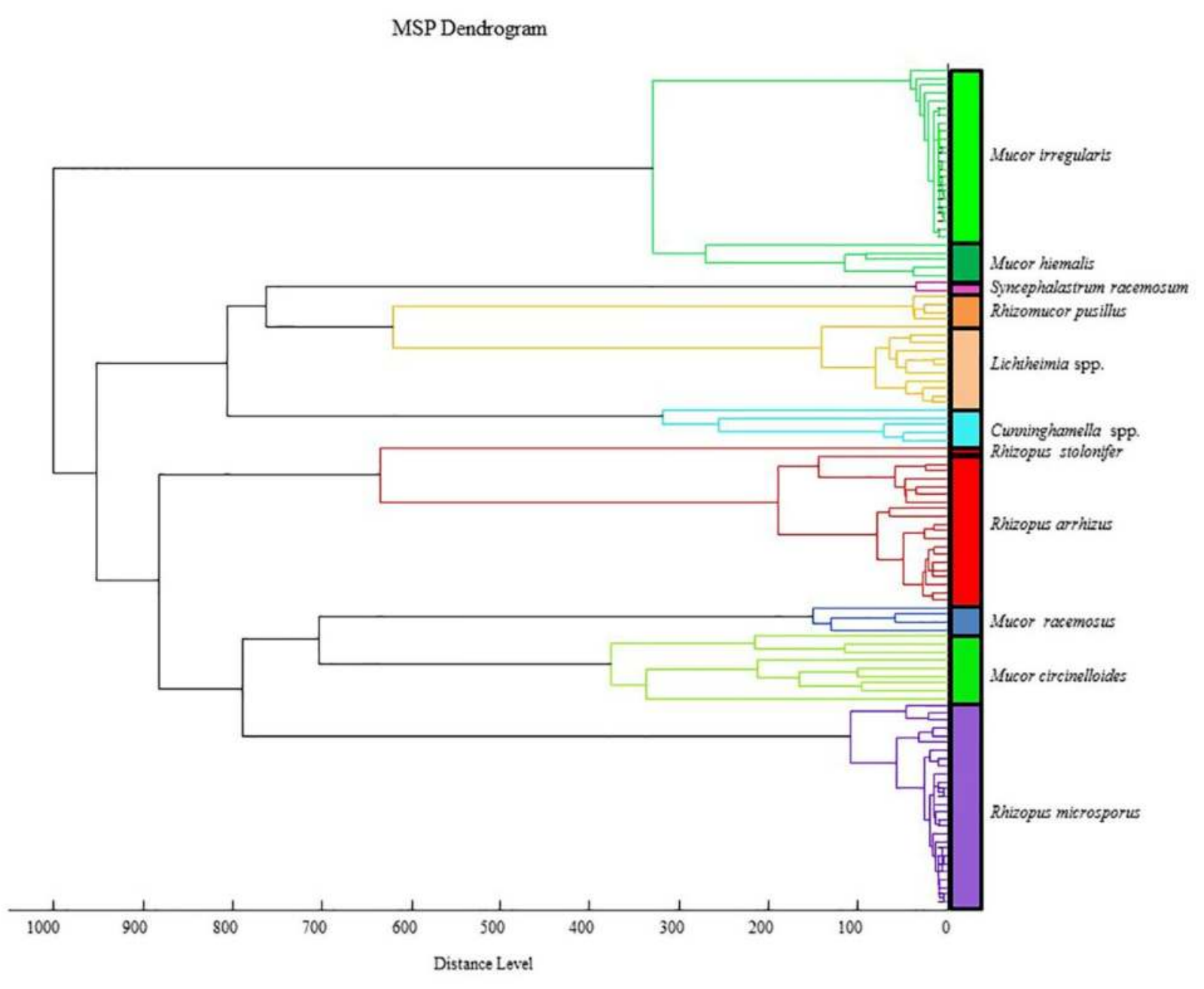

Fig. 1 Cluster analysis based on the main MSPs of all 111 isolates, which belong to 6 different clinically relevant genera of Mucorales. The distance levels of each subdivision are shown in arbitrary units, and the isolates of the Lichtheimia and Cunninghamella genera are shown as spp. identify $90(81.1 \%)$ and $111(100 \%)$ isolates at the species and genus levels, respectively, with a significantly increased accuracy rate. None of the 23 clinical strains of $M$. irregularis were reliably identified using only the Bruker library, whereas all isolates were correctly identified at the species level after the Bruker library and BMU database were combined. Phylogeny based on the internal transcribed spacer (ITS) sequences of Rhizopus spp. and Mucor spp. agreed well with main spectrum profile (MSP) dendrograms.

Conclusion: MALDI-TOF MS poorly identified Mucorales when the Bruker library was used alone due to its lack of some fungal species. In contrast, this technique perfectly identified $M$. irregularis after MSPs of relevant reference strains were added to the Bruker library. With an expanded Bruker library, MALDITOF MS is an effective tool for the identification of pathogenic Mucorales. Additionally, MSP dendrograms based on MALDITOF MS analysis were excellent for species discrimination.

Table 1 Identification of 111 clinical isolates by the Bruker library and the Bruker library plus BMU database

\begin{tabular}{|c|c|c|c|c|c|c|c|c|}
\hline \multirow{3}{*}{$\begin{array}{l}\text { Organism (no. of } \\
\text { isolates) }\end{array}$} & \multicolumn{8}{|c|}{ No. (\%) of isolates at the genus or species level identified by $\log ($ score) value } \\
\hline & \multicolumn{4}{|c|}{ Bruker library } & \multicolumn{4}{|c|}{ Bruker library plus BMU database } \\
\hline & $\geq 2.0$ & $\geq 1.7$ & $<1.7$ & Mis-ID & $\geq 2.0$ & $\geq 1.7$ & $<1.7$ & Mis-ID \\
\hline R. arrhizus (20) & $19(95)$ & $20(100)$ & $0(0)$ & $0(0)$ & $19(95)$ & $20(100)$ & $0(0)$ & $0(0)$ \\
\hline R. microsporus (27) & $24(88.9)$ & $27(100)$ & $0(0)$ & $0(0)$ & $24(88.9)$ & $27(100)$ & $0(0)$ & $0(0)$ \\
\hline R. stolonifer (1) & $0(0)$ & $1(100)$ & $0(0)$ & $0(0)$ & $0(0)$ & $1(100)$ & $0(0)$ & $0(0)$ \\
\hline R. pusillus (4) & $4(100)$ & $4(100)$ & $0(0)$ & $0(0)$ & $4(100)$ & $4(100)$ & $0(0)$ & $0(0)$ \\
\hline S. racemosum (2) & $2(100)$ & $2(100)$ & $0(0)$ & $0(0)$ & $2(100)$ & $2(100)$ & $0(0)$ & $0(0)$ \\
\hline L. corymbifera (4) & $4(100)$ & $4(100)$ & $0(0)$ & $0(0)$ & $4(100)$ & $4(100)$ & $0(0)$ & $0(0)$ \\
\hline L. ramosa (6) & $0(0)$ & $3(50)$ & $0(0)$ & $3(50)^{a}$ & $0(0)$ & $3(50)$ & $0(0)$ & $3(50)^{\mathrm{a}}$ \\
\hline L. ornata (1) & $0(0)$ & $0(0)$ & $0(0)$ & $1(100)^{b}$ & $0(0)$ & $0(0)$ & $0(0)$ & $1(100)^{\mathrm{b}}$ \\
\hline M. circinelloides $(9)$ & $2(22.2)$ & $6(66.7)$ & $0(0)$ & $3(33.3)^{\mathrm{c}}$ & $2(22.2)$ & $6(66.7)$ & $0(0)$ & $3(33.3)^{\mathrm{c}}$ \\
\hline M. irregularis (23) & $0(0)$ & $0(0)$ & $23(100)$ & $0(0)$ & $23(100)$ & $23(100)$ & $0(0)$ & $0(0)$ \\
\hline M. hiemalis (5) & $0(0)$ & $0(0)$ & $5(100)$ & $0(0)$ & $3(60)$ & $5(100)$ & $0(0)$ & $0(0)$ \\
\hline M. racemosus (4) & $0(0)$ & $0(0)$ & $4(100)$ & $0(0)$ & $4(100)$ & $4(100)$ & $0(0)$ & $0(0)$ \\
\hline C. bertholletiae (3) & $0(0)$ & $0(0)$ & $3(100)$ & $0(0)$ & $3(100)$ & $3(100)$ & $0(0)$ & $0(0)$ \\
\hline C. phaeospora (1) & $0(0)$ & $0(0)$ & $1(100)$ & $0(0)$ & $1(100)$ & $1(100)$ & $0(0)$ & $0(0)$ \\
\hline C. echinulata (1) & $0(0)$ & $0(0)$ & $1(100)$ & $0(0)$ & $1(100)$ & $1(100)$ & $0(0)$ & $0(0)$ \\
\hline Total (111) & $55(49.5)$ & $67(60.4)$ & $37(33.3)$ & $7(6.3)$ & $90(81.1)$ & $104(93.7)$ & $0(0)$ & $7(6.3)^{d}$ \\
\hline
\end{tabular}

Mis-ID, misidentification

${ }^{a}$ Misidentified as Lichtheimia corymbifera

${ }^{\mathrm{b}}$ Misidentified as Lichtheimia corymbifera

${ }^{\circ}$ Misidentified as Mucor ramosissimus

${ }^{\mathrm{d}}$ Misidentified at the species level but correctly identified at the genus level 